Analysis. - Weighed quantities of the crystals were decomposed with water in a Meyer-Jannek apparatus. ${ }^{3}$ The free selenic acid was reduced to selenious acid by heating with conc. hydrochloric acid, after which it was reduced to elementary selenium by hydrazine. Two analyses yielded 45.0 and $44.5 \%$ of selenium, while $\mathrm{SeO}_{2}(\mathrm{OH})(\mathrm{ONO})$ requires $45.3 \%$ of selenium.

For the determination of nitrogen, weighed quantities of the crystals were shaken in a closed bottle with a solution of sodiur. hydroxide until the nitrogen oxide fumes which were evolved had again been absorbed. The resulting solution was treated with a measured excess of potassium permangenate solution, and after oxidation of the nitrous to the nitric acid, the excess of permanganate was titrated back by means of oxalic acid. In two samples, 7.3 and $7.4 \%$ of nitrogen were found. Nitrosyl selenic acid requires 8 . of nitrogen. Considering the methods of analysis used, the results are sufficiently accurate.

Nitrosyl selenic acid, $\mathrm{SeO}_{2}(\mathrm{OH})(\mathrm{ONO})$, is decomposed by water. It is readily soluble in conc. selenic and conc. sulfuric acids. Nitrosyl sulfuric acid is also soluble in these acids, and both compounds are soluble in absolute alcohol but not in ether. At $80^{\circ}$, nitrosyl selenic acid melts with decomposition. This is also the melting point of nitrosyl sulfuric acid and is considerably higher than that of pure selenic acid, which is $58^{\circ}$.

Nitrosyl selenic acid is very reactive and with certain of the organic amines readily forms compounds, which we are studying.

BRESLAU, GERMANY

[CONTRIBUtion from the Kent Chemical Laboratory OF the UNiversity of Chicago]

\title{
THE SEPARATION OF ISOTOPES BY THERMAL AND PRESSURE
} DIFFUSION

\author{
By Robert S. MUlliken ${ }^{1}$ \\ Received March 1, 1922
}

\section{Introduction}

With the ultimate aim of obtaining extensive separations of isotopes, a careful preliminary study, both theoretical and experimental, is being made, in order to find the best practical method or methods. In a previous paper by Mulliken and Harkins ${ }^{2}$ the theory was developed and equations obtained for the change of composition and atomic weight for the fractions obtained when a mixture of isotopes is subjected to a process of irreversible evaporation, molecular effusion, molecular diffusion, or gaseous diffusion. A rather complete summary of the possible methods for separating isotopes was also given (p. 62). In the present paper, the theory of the method of thermal diffusion and that of the centrifugal

${ }^{3}$ Meyer and Jannek, ibid., 83, 51 (1913).

${ }^{1}$ National Research Fellow in Physical Chemistry.

2 Mulliken and Harkins, Thrs Jovrnar, 44, 37 (1922). 
method, as applied to the separation of isotopes, are rather fully discussed. Equations analogous to those for the other methods of separation are obtained, and used in a study of the applicability of the methods to various isotopic elements. Conclusions are reached as to the practical value of the two methods.

\section{Equations for Diffusion and Evaporation Processes}

The most important equations obtained in the previous paper will here be reviewed briefly, using the same equation numbers as there given.

For a mixture of two isotopes, the equations for the residue in an evaporation or diffusion process are, $\quad \Delta x_{2}=\frac{(1-k) x_{1} x_{2}}{2\left(x_{1}+k x_{2}\right)} \cdot \ln C=A \ln C$

or approximately,

$$
\Delta x_{2}=\frac{\left(M_{2}-M_{1}\right) x_{1} x_{2}}{2 M} \cdot \ln C
$$

and

$$
\begin{gathered}
\Delta M=A\left(M_{2}-M_{1}\right) \ln C=B \ln C \\
\Delta M=\frac{\left(M_{2}-M_{1}\right)^{2} x_{1} x_{2}}{2 M} \cdot \ln C
\end{gathered}
$$

Similar relations hold for a mixture of any number of isotopes. The $x$ 's denote mol-fractions, the $M$ 's atomic or molecular weights; $k=\sqrt{M_{1} / M_{2}}$; and $C$, the "cut," is the ratio of the initial to the final number of mols present in the residue. ${ }^{3}$ The subscript 1 refers to the lighter, 2 , to the heavier isotope. $M$ without subscript stands for the ordinary, average, atomic or molecular weight. $B$ is called the separation coefficient.

For the diffused or evaporated material, the équations are,

$$
\begin{gathered}
-\Delta x_{2}=A \ln C /(C-1) \\
\text { and }-\Delta M=B \ln C /(C-1)
\end{gathered}
$$

The total difference in atomic weight ${ }^{4}$ between the two fractions is then

$$
\begin{gathered}
\Delta \Delta M=\Delta M-(-\Delta M)=[B+B /(C-1)] \cdot \ln C=B \cdot C \cdot \ln C /(C-1) \\
=\frac{\left(M_{2}-M_{1}\right)^{2} x_{1} x_{2}}{2 M} \cdot \frac{C}{C-1} \ln C \text {, nearly }
\end{gathered}
$$

For $C=1, \Delta \Delta M=B$; for $C=2, \Delta \Delta M=2 B$ ln $2=1.386 B$; for $C=4, \Delta \Delta M=(8 / 3)$ $B \ln 2=1.848 B$; etc. The value for $C=2$ is of the most interest, since it corresponds to a division of the material into two equal fractions. All the above equations hold for a mixture of more than two isotopes, except that the expressions for $A$ and $B$ are more complex (see previous paper)

\section{Thermal Diffusion}

It has been shown theoretically ${ }^{5}$ and experimentally ${ }^{6,7}$ that if a gaseous

${ }^{3}$ In the case of gaseous diffusion, the numerical factor in the denominator is probably always greater than 2. This factor is equal to the exponent $c$ in the value of $k$ : in general, $k=\sqrt[c]{M_{1} / M_{2}}$.

4 This equation was not given in the previous paper.

${ }^{5}$ Enskog, Physik. Z, 12, 538 (1911); Ann. Physik, 38, 750 (1912); and Chapman, Phil. Trans., 217A, 115 (1916); Phil. Mag., [6] 34, 146 (1917).

${ }^{6}$ Chapman and Dootson, Phil, Mag., [6] 33, 248 (1917).

7 Ibbs, Proc. Roy. Soc, 99, 385 (1921). 
mixture is present in a container, one portion of which is kept hot, and another cold, an equilibrium state is attained in which there is an increased concentration of the larger or heavier molecules at the cold end, and vice versa. For a mixture of two isotopes, Chapman deduces ${ }^{8}$ the relation (here altered to conform to the present notation),

$$
\Delta_{i x_{2}}=k_{t} \text { in } T^{\prime} / T=(17 / 3) \cdot \frac{M_{2}-M_{1}}{M_{2}+M_{1}} \cdot \frac{x_{1} x_{2}}{9.15-8.25 x_{1} x_{2}} \cdot \ln T^{\prime} / T,
$$

for molecules which behave like elastic spheres, $\Delta_{t} x_{2}$ standing for the difference between the values of $x_{2}$ in the regions at the two temperatures. Actually, in the case of a mixture of hydrogen and carbon dioxide, $k_{t}$ proves to be only about $1 / 2$ or $1 / 3$ as large because the molecules do not behave as assumed. Approximately, since

$$
\Delta M=\left(M_{2}-M_{1}\right) \Delta x_{2}, \Delta_{l} M=K . \frac{\left(M_{2}-M_{1}\right)^{2} x_{1} x_{2}}{2 M_{2}} \cdot \ln T^{\prime} / T=K B \ln T^{\prime} / T
$$

The analogy to Equations $7 \mathrm{~A}$ and 19 is obvious. Except for some variability in $K$ for different types of molecules, the separation coefficient $K B$ for thermal diffusion depends in nearly the same way on the molfractions and molecular weights of the isotopes as does the coefficient $B$ for ordinary diffusion. Thermal diffusion is apparently however a much weaker agent than ordinary diffusion, under any practicable conditions, for if $K=1 / 4$, a reasonable estimate, then a temperature ratio of 15.5 (e. g., $80^{\circ}$ absolute against $1240^{\circ}$ absolute) will give $\Delta_{t} M=$ $(1 / 4) B \ln 15.5=0.693 B=B \ln 2$. Even if the mixture is divided almost wholly into two equal fractions at the two extreme temperatures, the value of $\Delta t M$ is only half as great as the corresponding value $(2 B \ln 2)$ of $\Delta \Delta M$ for a cut of 2 by one of the diffusion methods previously considered. This is in spite of the very large temperature ratio, corresponding to a range from liquid air temperature to $1000^{\circ}$. Evidently the method of thermal diffusion cannot compete ${ }^{9}$ with the other diffusion methods as a means of separating isotopes.

Evaporative Thermal Diffusion.-Probably the most favorable way to apply thermal diffusion would be to use a method of procedure similar to that proposed in the case of centrifugal separation, viz., to have a supply of the liquid mixture in the cold bulb, and to draw off gas very slowly from the hot bulb. The rate of separation ${ }^{10}$ would be the same as for an ordinary diffusion or an irreversible evaporation having a separation coefficient equal to $\Delta_{l} M$. As a matter of theoretical interest it is intended to test this method of "evaporative thermal diffusion" experimentally with mercury. If the process of drawing off the gas took place through a

s Chapman, Phil. Mag., [6] 38, 182 (1919).

9 "The method of thermal diffusion can, it is true, be adapted to rapid continuous operation, but probably only at a large sacrifice of efficiency (cf. Ibbs, Ref. 7 ).

' Compare discussion under centrifugal separation. 
porous wall, the effect of ordinary diffusion would be added to that of thermal diffusion, and the result would be the same as for an ordinary diffusion with a separation coefficient $\left(B+\Delta_{t} M\right)$, instead of $B$. This increase would, however, hardly be worth the added difficulties.

\section{Pressure Diffusion}

Development of Equations.--The problem of the separation of isotopes by "pressure diffusion," that is, by virtue of variation of composition along a pressure gradient, due either to a gravitational field or to centrifugal force, has been discussed by Lindemann and Aston, ${ }^{12}$ and by Chapman, ${ }^{8}$ who compares the method with that of thermal diffusion. I.indemann and Aston derive equations applicable to a gaseous mixture of two isotopes. These, slightly modified, and transcribed, are as follows. For gravitational separation, $r=e^{\frac{B \Delta h\left(M_{2}-M_{2}\right)}{R^{T}}}$

For gravitational separation, $r=e,(22)$ and for centrifugal separation $r=e^{\frac{v \cdot\left(M T_{2}-M M_{1}\right)}{2 R T}}$ (23). Here $r$ stands $^{12}$ for $\frac{x_{2} / x_{1}}{\left(x_{2}\right)_{0} /\left(x_{1}\right)_{0}}$, the subscript 0 referring to the point of reference, for example, the center of the centrifuge, or the level of the earth; $v$ is the peripheral velocity. Chapman gives the equation (here transcribed), applicable to any type of pressure diffusion,

$$
J_{p} x_{2}=k_{p} \ln p / p_{0}=\frac{\left(M_{2}-M_{1}\right) x_{1} x_{2}}{M_{1} x_{1}+M_{2} x_{2}} \cdot \ln p / p_{0} .
$$

analogous to his equation for thermal diffusion.

Equations can be derived for the case of pressure diffusion, analogous to those for other types of diffusion, which show clearly the factors on which the change of atomic weight depends. It will be best to go through with the derivation from the beginning, since the methods and notation are different from those of Lindemann and Aston, and of Chapman. For a gaseous mixture of two isotopes at total pressure $p$ and density $\rho$, subjected to centrifugal action, $d p_{1}=\frac{v^{2} \rho_{1} \mathrm{~d} r}{r}=\rho_{1} \omega^{2} r \mathrm{~d} r=\frac{M_{1} p_{1} \omega^{2} r \mathrm{~d} r}{R T}$. Here $p_{1}$ and $\rho_{1}$ refer to the partial pressure and density of the lighter isotope, and $v, \omega$, and $r$, denote respectively linear velocity, angular velocity, and radial distance. An analogous equation holds for the heavier isotope. The above equation gives $\frac{\mathrm{d} p_{1}}{p_{1}}=\mathrm{d} \ln p_{1}=\frac{M_{1} \omega^{2}}{R T} r \mathrm{~d} r$. Then

$$
\frac{\ln p_{1}}{\left(p_{1}\right)_{0}}=\frac{M_{1} w^{2}\left(r^{2}-r_{0}^{2}\right)}{2 R T}=\frac{M_{1}\left(v^{2}-v_{\theta}^{2}\right)}{2 R T} \text {. }
$$

${ }^{11}$ Lindemann and Aston, Phil. Mag., [6] 37, 523 (1919).

12 The quantity $r$ is analogous to the "enrichment ratio" of Rayleigh (see Mulliken and Harkins, Ref. 2), but differs in that it corresponds to $\Delta_{p} M$ instead of to $\Delta M$ 
If $\gamma_{0}=0, v_{0}=0$. By combining Equation 25 with the corresponding expression in terms of $p_{2}$, and putting $v_{0}=0$, Lindemann and Aston's relation (23) can be obtained. The following relation, which will be useful in another connection, can also be seen to hold approximately,

$$
\ln \frac{\dot{p}^{\prime}}{p, 1}=\frac{M\left(v^{2}-v_{0}^{2}\right)}{2 R T} \text {. }
$$

Chapman's relation (24) for $\Delta_{p} x_{2}$ can be obtained by a derivation analogous to that used in the paper of Mulliken and Harkins in arriving at Equations 2, 5, and 6. Thus from the equations above given for $\mathrm{d} p_{1} / p_{1}$ and $\mathrm{d} p_{2} / p_{2}, \mathrm{~d} p_{2} / p_{2}=\left(M_{2} / M_{1}\right) \cdot \mathrm{d} p_{1} / p_{1}$. Then since $p_{1}=p x_{1}$ and $p_{2}=p x_{2}$, we have $\frac{p \mathrm{~d} x_{2}+x_{2} \mathrm{~d} p}{p x_{2}}=\frac{M_{2}}{M_{1}} \cdot \frac{p \mathrm{~d} x_{1}+x_{1} \mathrm{~d} p}{p x_{1}}$. From this point the derivation is precisely analogous to that used in obtaining Equation 6 of the previous paper, everything being unchanged except that $p$ everywhere replaces $N\left(p_{0} / p\right.$ replaces $\left.N_{0} / N=C\right)$, and $M_{2} / M_{1}$ replaces $\sqrt[c]{M_{1} / M_{2}}$ $=k$. Thus we obtain the relation, formally analogous ${ }^{13}$ to Equation 6 ,

$$
\Delta_{p} x_{2}=\frac{\left(1-M_{2} / M_{1}\right) x_{1} x_{2}}{x_{1}+\left(M_{2} / M_{1}\right) x_{2}} \cdot \ln \frac{p_{0}}{p}
$$

Then

$$
\Delta_{p} M=\left(M_{2}-M_{1}\right) \Delta_{1}, x_{2}=\frac{\left(M_{2}-M_{1}\right)^{2} x_{1} x_{2}}{M} \cdot \ln \frac{p}{p_{0}}
$$

which is formally analogous ${ }^{13}$ to Equations 7 and $7 \mathrm{~A}$. Combining (26) and (27), there results the approximate relation ${ }^{14}$ for the difference in atomic weight at points $r$ and $r_{0}$

$$
\Delta_{p} M=\frac{\left(M_{2}-M_{1}\right)^{2} x_{1} x_{2}\left(v^{2}-v_{0}^{2}\right)}{2 R T}=P\left(v^{2}-v_{0}^{2}\right)=P\left(\omega^{2}\left(r^{2}-r_{0}^{2}\right) .\right.
$$

For the general case of a mixture of several (atomic, or molecular) isotopes, the corresponding relation ${ }^{15}$ is $\Delta_{p} M=\frac{\Sigma\left(M_{a}^{2} x_{a}\right)-\left[\Sigma\left(M_{a} x_{a}\right)\right]^{2}}{2 R T}$.

${ }^{13} \Delta_{p} x_{2}$ and $\Delta_{p} M$ are really more nearly analogous to $\Delta \Delta x_{2}$ and $\Delta \Delta M$ (compare Equation 19), in physical meaning, than to $\Delta x_{2}$ and $\Delta M$.

14 Similarly, for gravitational separation, which is of course not a practical method, $\Delta_{f} M=\left(M_{2}-M_{1}\right)^{2} x_{1} x_{2} g\left(h-h_{0}\right) / R T=2 P^{\prime} g \Delta h(29)$.

${ }^{15}$ This is obtained as follows. Aralogons to Equation $6^{\prime}$ of the previous paper, we have here $\lambda_{p} x_{a}=\frac{x_{a}\left(S-k_{a}^{\prime}\right)}{S}$. In $p_{0} / p$. Here $k_{a}^{\prime}=M_{a} / M_{1}$, and $S=\Sigma x_{a} k^{\prime}{ }_{a}=$ $\frac{\Sigma x_{a} M_{a}}{M_{1}}=\frac{M}{M_{1}}$ and $S-k_{a}^{\prime}=\left(M-M_{a}\right) / M_{1}$. So $\Delta_{p} x_{a}=\frac{x_{a}\left(M_{a}-M\right)}{M}$. $\ln \frac{p}{p_{0}}=$ $\frac{x_{a}\left(M_{a}-M\right)\left(v^{2}-v_{0}^{2}\right)}{2 R T} \cdot$ Now $\lrcorner_{p} M=\Sigma\left(M_{a}-M_{1}\right) \perp x_{a}=\frac{v^{2}-v_{a}^{2}}{2 R T} . \Sigma\left[x_{a}\left(M_{a}-M\right)\left(M_{a}-M_{1}\right)\right]$.

The summation can be expanded to $\Sigma x_{a} M_{a}{ }^{2}-M \Sigma x_{a} M_{a}-M_{1} \Sigma x_{a} M_{a}+M M_{1} \Sigma x_{a}=$ 


$$
\begin{aligned}
& \left(v^{2}-v_{0}^{2}\right)=\frac{\Sigma\left(M_{a}^{2} x_{a}\right)-M^{2}}{2 R T} \cdot\left(v^{2}-v_{0}^{2}\right)=\frac{\bar{M}^{2}-(\bar{M})^{2}}{2 R T}\left(v^{2}-v_{0}^{2}\right)=\frac{\Sigma_{a} \Sigma_{b} x_{a} x_{b}\left(M_{a}-M_{b}\right)^{2}}{2 R T} . \\
& \left(v^{2}-v_{0}^{2}\right)=P^{\prime}\left(v^{2}-v_{0}^{2}\right) .
\end{aligned}
$$

The subscripts $a$ and $b$ here refer independently to each isotope in turn. ${ }^{16}$ In the last expression, each like term of the summation is to be taken only once (in the complete summation each appears twice). The expression for $P$, although different in form from that for $P^{\prime}$, is obtainable from the latter as a special case. ${ }^{17}$ The only approximations in deriving the expressions for $P$ and $P^{\prime}$ are those involved in the use of Equation 26, and that of using a differential quantity $\mathrm{d} x_{a}$ as a finite increment $\Delta_{p} x_{a}$. The error involved is only a few per cent. unless $\Delta_{p} M$ is large, in which case the change of $P$ and $P^{\prime}$ with $M$ can be allowed for by inserting a term obtained by taking the second derivative. (Compare discussion and Equations 9 and 10 of the previous paper.)

Comparison of Centrifugal and Ordinary Separation Methods and Coefficients.-The following values of the "centrifugal separation coefficients" ( $P$ or $\left.P^{\prime}\right)$ have been calculated for several elements at $20^{\circ}$ : $\mathrm{I}, \mathrm{i}, 1.16 \times 10^{-12} ; \mathrm{B}, 1.85 \times 10^{-12} ; \mathrm{Ne}, 7.38 \times 10^{-12} ; \mathrm{Mg}, 8.97 \times$ $10^{-12} ; \mathrm{Cl}, 14.5 \times 10^{-12} ; \mathrm{Ni}, 18.4 \times 10^{-12} ; \mathrm{Zn}, 53 \times 10^{-12} ; \mathrm{Br}$, $20.4 \times 10^{-12} ; \mathrm{Hg}, 47 \times 10^{-12}$. For ordinary air, the coefficient would be about $62 \times 10^{-12}$. The values for most of the even-numbered heavy elements (beginning with zinc) are doubtless high, like those for zinc and mercury. The values have been calculated chiefly from atomic weight and positive-ray analysis data ${ }^{18}$ in the case of mercury, the value has been calculated from the approximate relation $P^{\prime}=M / R T . B$, using the experimental value of the ordinary (diffusion) separation coefficient $B$ obtained by Mulliken and Harkins. An important feature of the centrifugal separation coefficient which differentiates it from the ordinary sepa-

$$
\begin{aligned}
& \Sigma x_{a} M_{a^{2}}-M^{2} \text {, since } \Sigma x_{a} M_{a}=M, \text { and } \Sigma x_{a}=1 . \text { Then } \Delta_{p} M=\frac{\Sigma\left(M_{a}{ }^{2} x_{a}\right)-M^{2}}{2 R T} . \quad\left(v^{2}-v_{a}^{2}\right)= \\
& P^{\prime}\left(v^{2}-v_{0}{ }^{2}\right)
\end{aligned}
$$

The last expression in the text for $P^{\prime}$ of Equation $28^{\prime}$ readily reduces, on expansion, to the form just obtained. $P$ and $P^{\prime}$ are related to $E$ of Equation $7 A$ and $E^{\prime}$ of Equation $7 \mathrm{~A}^{\prime}$ of the previous paper. $E=\frac{\left(M_{2}-M_{1}\right)^{2} x_{1} x_{2}}{c M_{2}}=\frac{2 R T}{c M_{2}} \cdot P=\frac{R T}{M_{2}} \cdot P$, if $c=2$; and $E^{\prime}=\frac{\Sigma x_{a} x_{b}\left(M_{a}-M_{b}\right)^{2}}{c M}=\frac{2 R T}{c M} \cdot P^{\prime}=\frac{R T}{M} \cdot P^{\prime}$, if $c=2$.

${ }^{16}$ For gravitational separation, the expression corresponding to Equation $28^{\prime}$ is \lrcorner$_{p} M=2 P^{\prime}, \mathrm{g} \Delta h$.

${ }^{17}$ Similarly, $E$ could have been obtained from $E^{\prime}$ as a special case, in the previous paper.

18 The value for zinc is based on the existence of four isotopes $64,66,68$, and 70 , as recently reported by Dempster. 
ration coefficient, is that it is independent ${ }^{19}$ of the state of combination of the element, ${ }^{20}$ and is thus characteristic of the latter. This is true for each element even in compounds containing more than one isotopic element. ${ }^{21}$ The ordinary separation coefficient for a given element is in-

${ }^{10}$ If the molecules are built up from the atoms according to chance, the mol-fractions of the various molecular isotopes are given by the successive terms of the expansion of $\left(x_{1}+x_{2}+\ldots x_{s}\right)^{n} e$, where $x_{1} x_{2}, \ldots$, refer to the atomic isotopes (see the previous paper for derivation of this). On this basis, the exact constancy of $P^{\prime} / n_{e}$ has been tested by comparison of the algebraic expressions for $n_{e}=1,2,3$, and 4 , for a mixture of two isotopic atomic species, and by calculation of numerical examples for $n_{\theta}=1$ and 2 , for the case of a mixture of three atomic isotopes. ( $P^{\prime}$ is here the molecular separation coefficient, $n_{\theta}$ the number of atoms of the element per molecule). Although no general proof has been obtained, it is probable that all possible expressions for $P^{\prime} / n_{e}$ for various compounds of an element containing any' number of isotopes, can be reduced to a common expression, the same as that for the case $n_{\theta}=1$. Mathematically, the relation $P^{\prime} / n_{\theta}=$ $\frac{\Sigma\left(M r_{b}^{2} x_{b}\right)-M^{2}}{n_{e}}=\Sigma\left(A_{a} x_{a}\right)-A^{2}$ would have to hold for all compounds and all values of $n_{0}$. For a mixture of $i w o$ isotopes, this reduces to $\left(A_{2}-A_{1}\right)^{2} x_{1} x_{2}(A$ and $M$ stand for atomic and molecular weights respectivaly, and the subscripts $a$ and $b$ refer respectively to atomic and molecular species). The existence of this relation no doubt springs from an absence of dependence of the value of $\Delta x_{a}$ (for any given atomic isotope in a given operation) on the manner of distribution of the atomic species $a$ among the various molecular isotopes present. Apparently the assumed distribution, $i . e$, according to chance, is such as always to yield the same result, so that the change of atomic weight, $\triangle A=\mathbb{Z}\left(A_{a}-A_{1}\right) \Delta x_{a}$, is independent of the particular compound in which the isotopic element occurs.

20 Ordinarily the term "separation coefficient" is applied to the change of atomic weight of the element concerned. It could also be applied to the coefficients $B, P$, etc., for molecules, for the change of moleculct weight. In the case of centrifugal separation, the molecular coefficient is eciual to the sum of the products of the atomic coefficients by the numbers of atoms of the corresponding elements in the molecule.

21 Suppose a compound of molecular weight $M$ contains 2 or more isotopic elements. Let us divide $M$ into 2 parts, such that $M=X+Y$. Let $X$ stand for the partial molecuhar weight in the compound of the paticular isotopic element which we are interested in. $X$ will then be the atomic weight of this element times the number of atoms, $n_{e}$, which it contributes to each molecule. I.et $Y$ stand for the part of the molecular weight due to all other clements, some of which may be isotopic. $M$ is really an average value for a large number of molecular isotopes; $X$ and $Y$ likewise are average values. The isotopes of $M$ can be regarcied as consisting of all possible combinations of the isotopic forms $X_{1}, X_{2}$, etc., of the partiai molecule $X$, with the isotopic forms $Y_{1}, Y_{2}$, etc., of the partial molecule $Y$. The isotopes of $M I$ can then be designated by double subseripts, the first to denote the $X$ isotope, the second to denote the $Y$ isotope. Thus $M_{24}=X_{2}+$ $I_{i}$. We may also wish to deal with an average value of $Y$, while considering the $X$ isotopes individually, or aice rersa. Thus let $M_{20}$ represent $X_{2}+Y ; M_{03}, X+Y_{3}$, and so otl.

Equation $28^{\prime}$ shows no dependence of $P^{\prime}$ on the absolute value of $M$, so that if $Y$ had only a single value (no isotopes), the (molecular) separation coefficient $P^{\prime}$ (see Ref. 20) corresponding to $W$ would not be affected by the magnitude of $Y$, but would depend only on the isotopic composition of the partial molecule $X$. That is, $P^{\prime}$ would be the same whatever the value of $Y$, whether $Y_{1}, Y_{2}$, or some other value. If we consider the 
versely proportional to the molecular weight of the compound in which it appears, but is otherwise independent of the state of combination ${ }^{22,23}$ (i.e., of the number of its atoms per molecule or the presence of other isotopic elements). Due to this mass factor, the ordinary coefficient tends to fall with increasing atomic weight of the isotopic element (this tendency is largely balanced by the increasing spread of the atomic weights of the isotopes), whereas the centrifugal separation coefficient is not so affected. Centrifugal separation is therefore relatively much more favorable to the heavy elements, as well as absolutely due to the increased number of isotopes. The effect of the atomic weight differences and of the mol-fractions of $X$ partial molecules collectively, they can be divided into several groups of identical composition, distinguished by being combined with different $Y$ 's. Of all the molecules present, the fraction $y_{1}$ will be in the group $X Y_{1}, y_{2}$ in $X Y_{2}$, etc. Since for every fraction by itself, the separation coefficient would have the value $P^{\prime}$, the same must be true of the substance as a whole. That is, the value of $P^{\prime}$ is independent of the isotopic composition of the $Y$ part of the molecule. The correctness of this result is not affected by the fact that the mol-fractions $y_{1}, y_{2}$, etc, change during the diffusion due to the separation of the isotopes in the $Y$ part of the molecular weight, for the reasoning above holds at any instant, whatever the relative proportions of the different $Y$ groups (the coefficient $P^{\prime}$ is in its derivation an instantaneous quantity). The elementary coeffcient $P^{\prime} / n_{\theta}$ has already been shown with reasonable certainty (Ref. 19) to be independent of $n_{e}$, so that is now shown to be altogether independent of the state of combination of the element. Thus separation proceeds, for each isotopic element in a mixed molecule, independently of the other elements present.

The discussion above can be put in a mathematical form which will be useful as a basis for Ref. 23. Suppose the separation coefficient $P^{\prime}$ for any $Y$ group were not necessarily independent of the nature of the latter. Then the actual separation coefficient would be an average over all the different $Y$ groups. Denoting by $P_{1}^{\prime}, P_{2}^{\prime}$, etc., the several values of the separation coefficient corresponding to $Y_{1}, Y_{2}$, etc., we would have $P^{\prime}=P^{\prime}{ }_{1} y_{1}+P^{\prime} y_{2}+\ldots=(1 / 2 / R T) \quad\left[y_{1} \Sigma x_{a} x_{b}\left(M_{a 1}-M_{b_{1}}\right)^{2}+y_{2} \Sigma x_{a} x_{b}\left(M_{a 2}-M_{b_{2}}\right)^{2}\right.$ $+\ldots$. . But here $M_{a 1}-M_{a 2}=M_{a 2}-M_{b_{2}}=X_{a}-X_{b}$, that is, the effects of the different $Y^{\prime}$ s cancel, and the result is $P^{\prime}=\frac{\Sigma x_{a} \cdot x_{b}\left(X_{a}-X_{b}\right)^{2}}{2 R T,}$, the same as if a single average value, or the value zero, were used for $Y$; at the same time it is seen that $P_{1}^{\prime}=P^{\prime}{ }_{2}=\ldots$.

${ }_{22}$ The discussion in Ref. 19 applies to the values of the approximate diffusion separation coefficient $E^{\prime} / n_{e}$ (which is proportional to $P^{\prime} / n_{e}$ ), except that $E^{\prime} / n_{e}$ is inversely proportional to the total molecular weight: $E^{\prime}=\frac{2 R T}{c}, \frac{P^{\prime}}{M}$. It also applies approximately to the exact coefficient $B$ or $B^{\prime} / n_{e}$, holding usually to within 1 or $2 \%$ for the latter. These relations were stated in the previous paper but not as fully discussed as here.

${ }^{23}$ In the case of the ordinary separation coefficient we have analogous to the above discussion in Note $21, E^{\prime} n_{e}=\frac{1}{c n_{e}}\left[\frac{y_{1} \Sigma x_{a} x_{b}\left(X_{a}-X_{b}\right)^{2}}{M_{01}}+\frac{y_{2} \Sigma x_{a} x_{b}\left(X_{a}-X_{b}\right)^{2}}{M_{02}}+\ldots\right]$ $=\frac{\Sigma x_{a} x_{b}\left(X_{a}-X_{b}\right)^{2}}{c n_{e}}\left[\frac{y_{1}}{M_{01}}+\frac{y_{2}}{M_{02}}+--\right]=\frac{\Sigma x_{a} x_{b}\left(X_{a}-X_{b}\right)^{2}}{c n_{e} M}$, nearly, $=\frac{2 R T}{c M} .\left(P^{\prime} / n_{e}\right)$.

These relations of course hold also for $E\left(n_{e}=1\right)$, and for $B$ and $B^{\prime} / n_{e}$; in all cases approximations are involved, but these are usually close. 
the various isotopes of a given element, is the same for both the ordinary and the centrifugal separation coefficients (also for the thermal diffusion coefficient); they differ in the dependence of the former (the same is true of the thermal coefficient) on the magnitude of the atomic (or molecular) weight.

In a centrifugal separation, the degree of separation varies continuously with the distance from the axis of the apparatus, as expressed by Equation 28 or $28^{\prime}$. In using Equations 23 and 28 or $28^{\prime}$ it should be remembered that $\Delta_{p} M$ is the difference in atomic weight between material in different regions. The absolute atomic weights of any fractions depend on the distribution of material in the centrifuge. The only generalization which can be made is that the original or average atomic weight must be somewhere between the extremes at center and periphery. If the material were largely concentrated in the periphery, the decrease of atomic weight would be nearly $\Delta_{p} M$ for the light fraction, while the increase would be only slight for the denser fraction. Note that $\Delta_{p} M$ varies as the square of the angular velocity, and also as the square of the radius. $\Delta_{p} M$ also varies inversely as the absolute temperature.

It is of interest to calculate the peripheral velocity $v$ (or in general the value of $\sqrt{\left.v^{2}-v_{0}^{2}\right)}$ necessary to produce a difference of atomic weight $\Delta_{p} M$ equal to that secured in an ordinary diffusion or evaporation with a cut of 2. For the latter case, $\Delta \Delta M=2 B \ln 2$; for the former, $\Delta_{p} M$ $=\frac{M}{R T} \cdot \mathrm{B} \cdot v^{2}$, nearly. Equating $\Delta_{p} M$ and $\Delta \Delta M$, it is found that $v^{2}=$ 1. $\frac{386 R T}{M}$. This gives $v=\frac{181,000}{\sqrt{M}}$ for $t=20^{\circ} \mathrm{C}$. For $M=6.94(\mathrm{~L}, \mathrm{i})$, $v=6.9 \times 10^{4} ;$ for $M=36.46(\mathrm{HC} 1), v=3.0 \times 10^{4} ;$ for $M=200.6$ $(\mathrm{Hg}), v=1.28 \times 10^{4}$. If the ratio of the value of $\Delta_{p} M$ for $v=10^{5}$ to that of $\Delta \Delta M$ for a cut of 2 is calculated the values obtained are, 2.2 for $\mathrm{I}, \mathrm{i}, 11.1$ for $\mathrm{HCl}, 61$ for $\mathrm{Hg}$. If $v=10^{4}$, the ratios are, $0.022,0.111$, and 0.61 . The value of the centrifugal method evidently depends on the possibility of obtaining and using a velocity approaching $10^{5} \mathrm{~cm} . /$ sec. If this can be done, the centrifugal method is clearly superior in theory to any other method for the heavier elements. The method has additional superiority in the fact that the separation should be just as great for any compound of an element, as already pointed out. There are, however, a number of difficulties, especially for the heavier elements, aside from that of obtaining the necessary speed.

Drawbacks to Centrifugal Method.-Among the factors that reduce the apparent advantages of the application of the centrifugal method to the separation of gaseous isotopic mixtures are (1) the difficulty of constructing a centrifuge which could consistently turn out separated products 
at as great a rate as a diffusion or evaporation apparatus; (2) the fact that the value of $\Delta p M$ depends on $\left(v^{2}-v_{0}^{2}\right)$, not on $v^{2}$ alone; (3) the necessity for removing the products continuously while the centrifuge is moving at full speed; (4) the fact that $\Delta_{p} M$ represents the extreme separation, and that it will be difficult to design an apparatus, continuous or otherwise, that will separate the input material at all completely into two more or less equal extreme fractions, especially in view of the fact that (5) at high speed a gas will very largely condense to a liquid, or become highly compressed, close to the periphery, so that the light fraction will be extremely small. In connection with (4), the average value of $\Delta_{p} M$, referred to the composition at the point $r_{0}$ nearest the center of the centrifuge, for the material between any two radial distances $r_{1}$ and $r_{2}$ from the center, is

$$
\begin{aligned}
& \left(\Delta_{p} M\right){ }_{r_{1}}^{r^{2}}=\frac{\left.\int_{r_{1}}^{r^{2}}(\lrcorner_{p} M\right)_{r} \mathrm{~d} r}{r_{2}-r_{1}}=\frac{P \omega^{2} \int_{r_{1}}^{r^{2}}\left(r^{2}-r_{0}{ }^{2}\right) \mathrm{d} r}{r_{2}-r_{1}}= \\
& P \omega^{2}\left[\frac{\left(r_{2}{ }^{3}-r_{1}^{3}\right) / 3-r_{0}^{2}\left(r_{2}-r_{1}\right)}{r_{2}-r_{1}}\right]=1 / 3 P_{\omega^{2}}\left(r_{2}^{2}+r_{1} r_{2}+r_{1}^{2}-3 r_{0}^{2}\right) .
\end{aligned}
$$

This is accurate only if the density is practically constant, and so is applicable especially to liquids. If the whole material is divided into two equal fractions by volume, then for a whirling cylindrical tube, or for a hollow disc, the actual separation, or difference in atomic weight, will be about half the extreme $\Delta_{p} M$. It can be shown that in such a case the departure of the denser fraction from the original or average composition is greater than that of the less dense fraction. If only extreme fractions are taken, the larger separation is at the expense of quantity. If the value of $\Delta_{p} M$ is large, there will be a large density-gradient (Factor 5 ), which will again reduce the quantity of the light fraction. The ratio of total pressure from periphery to center can be calculated from Equation 26

$$
\ln p / p_{0}=\frac{M\left(v^{2}-v_{0}^{2}\right)}{2 R T}=\frac{M}{P} \cdot \frac{\Delta_{p} M}{2 R T}=\frac{\Delta_{p} M}{2 B},
$$

$P$ (or $P^{\prime}$ ) being given by Equation 28 or $28^{\prime}$. For $\Delta_{p} M=1.386 B=$ $(\Delta \Delta M)_{c=2}$, we found $v=\frac{181,000}{\sqrt{M}}$, and so $\ln p / p_{0}=\frac{(181,000)^{2}}{2 R T}$, or $p / p_{0}$ $=e^{0.687}=2.0$. This pressure ratio is not large, but neither are the values of $\Delta_{p} M$. For large values of $\Delta_{p} M$, where the centrifugal method would be really worth while, the pressure ratio becomes large, and the difficulty of obtaining any appreciable quantity of separated product is greatly increased. Thus for $\Delta_{p} M=13.86 \mathrm{~B}, p / p_{0}=(2)^{10}=10^{3}$. For the high pressure ratios, the concentration of material near the periphery will be even more marked, due to departure from the behavior of a perfect gas. These difficulties cannot well be avoided by any special method of design.- 
Note that from (31), $p / p_{0}=e^{\Delta} w / 2 R$. This means that insofar as the value of $p / p_{0}$ sets a practical limit to $\Delta_{p} M$, the coefficient $B$, instead of $P$, is important, so that the heavy elements lose their advantage. On the whole, it seems probable that the direct separation of a gas or vapor into fractions by centrifuging would have no practical superiority, as a means of separating isotopes, over the diffusion or evaporation methods.

Method of Evaporative Centrifuging.- The following special adaptation of the centrifugal method seems rather promising as a means of securing a fairly large separation it a single operation in the case of certain grases. It should give greater separation than the method of dividing a gas directly into fractions, as well as being largely independent of the difficulties caused by large pressure ratios. For this purpose, the apparatus should have a considerable capacity near the periphery, which should be in free communication with the center, so that equilibration would be rapid. The gaseous iscitopic mixture to be centrifuged would be admitted through a tube connected with the center of the centrifuge. As the latter speeded up, more and more gas would be drawn in, and compressed or condensed in the periphery. When equilibrium had been established, under conditions such that nearly all the gas was concentrated in the periphery, the gas would be drawn off very slowly by reducing its pressure at the center of the apparatus. Any desired cut could be made, and the process would be analogous in its results to, although entirely different in mechanism from, a process of irreversible evaporation having a separation coefficient equal to the value of $\Delta_{p} M$, which represents difference in atomic weight between center and periphery. Gas thus drawn off corresponds to the "instantaneous condensate" in an evaporation. For the residue, in the periphery, the increase in atomic weight would be

$$
\left.(\Delta M)_{p}=\right\lrcorner_{p} . M \ln C=P_{v^{2}} \ln C ;
$$

for the gas drawn off,

$$
(-\Delta M)_{p}=\frac{\Delta_{p} M \ln C}{C-1}=\frac{P v^{2} \ln C}{C-1}
$$

and the total difference in atomic weight between the two fractions would be

$$
(\Delta M)_{p}=\frac{P_{v^{2}} C \ln C}{C-1} \text {. }
$$

'These equations are analogous to (7), (16), and (19). For a cut of 2, $(\Delta \Delta M)_{p}=1.386 P v^{2}$. In this last case, two separated fractions differing by $1.386 \mathrm{Pv}^{2}$ would be obtained; whereas, by merely splitting the gas in a centrifuge at the same speed into two fractions, even if the density of the gas could be uniform, the difference in average composition of the two fractions would be only $P v^{2} / 2$ units of atomic weight. The modified method thus should give a much larger practical separation, even aside from the question of the pressure ratio effect. Further, the product can be taken 
off in several fractions, if desired, and a large cut can be made on the residue in one operation, greatly increasing the separation. The method thus strongly resembles the evaporation method, and may be called "evaporative centrifuging." In practice, the efficiency of the method will be reduced somewhat (1) by the very fact that not all the gas will be in the periphery initially, and (2) by the disturbance of equilibrium caused by the drawing off of the gas. For the successful operation of the method of evaporative centrifuging, the speed and quantity of material used must be so adjusted that the gas pressure at the center will be great enough to handle, while the material in the periphery is, preferably, in the liquid state. This condition can be fulfilled, up to fairly high peripheral velocities, by a few gases of high critical pressure and low boiling point, such as hydrogen chloride, bromide, selenide, telluride and silicide. Thus for hydrogen chloride at $v=10^{5},(\Delta \Delta M)_{p}$, for a cut of 2 , would be 15 times as great as $\Delta \Delta M$ for a diffusion or evaporation. If the hydrogen chloride behaved like a perfect gas, the pressure ratio (about 2200) would correspond to a range from $15 \mathrm{~mm}$. to 4.5 atmospheres, the latter being approximately the vapor pressure of hydrogen chloride at $20^{\circ}$ (the critical pressure is 86 atmospheres).

General Considerations Respecting the Centrifuging of a Gas.For the lightest elements, the centrifugal method has no great theoretical superiority over the diffusion methods in degree of separation even for $v=10^{5}$. For the heavier elements or compounds, the pressure ratio becomes excessive at velocities too low to yield a very great separation. For gases of low critical pressure, the pressure ratio again limits the separation. For liquids, or gases of high critical temperature, heating is required (note that the degree of separation is inversely proportional to the absolute temperature). Thus the method of evaporative centrifuging is restricted in its usefulness to some of the elements of medium atomic weight. Here a separation 10-15 times as great as that obtainable by diffusion methods can be hoped for in a single operation. A greater separation than this in a single operation can hardly be hoped for under any practicable conditions.

Factors of Importance in Separating Isotopes by the Centrifuging of a Liquid.-As far as theory is concerned, a very large separation might be expected in the centrifuging of liquid elements of high atomic weight. One great advantage of such a method would be the ease with which the material could be divided into fractions, the difficulties caused by compression and condensation in the case of gases at high pressure ratios being practically absent. Equation 30 would apply quite well to the fractions obtained in centrifuging a liquid. New difficulties, however, enter if one attempts to obtain the largest separations. Thus for $v=$ $10^{5} \mathrm{~cm}$. per sec., $\Delta_{p} M$ Iis 60 times as great for mercury as is $\Delta \Delta M$ for a cut 
of 2 by a diffusion; but under these conditions the calculated pressure in the peripheral portion of the centrifuge would be 70,000 atmospheres, ${ }^{24}$ and it is found that the degree of separation is directly proportional to the peripheral pressure. Thus this factor invclves enormous, if not insuperable difficulties of construction for very large separations. The use of a suitable liquid compound of a heavy element of lower density would of course improve matters; and the use of: an aqueous solution of a salt has possibilities (several difficulties might come up here; also, the capacity of the apparatus would be greatly reiuced). The most important practical difficulty, as is shown by experinental evidence given below, is the effect of even slight vibration in nullifying the tendency toward separation. ${ }^{25}$ This may or may not be insuperable. Another practical factor to be considered is the rate of attainment of equilibrium, but the conditions are favorable in this respect. The evidence for the correctness of the theory in the case of a liquid seems practically as conclusive as for the case of a gas.

Theory of Separation of Isotopes by Liquid Centrifuging.-Lindemann and Aston ${ }^{11}$ give for the separation of a liquid into isotopes by centrifuging the same equation as for a gas. In connection with a discussion of the possibility of separating liquid mercury by this method, Poole ${ }^{26}$ gives a detailed derivation which would lead to equations identical with those of Lindemann and Aston, although Poole does not make the necessary final step. The equations in the present paper would then also hold. In Poole's derivation, he assumes that the buoyancy effect caused by the relative centrifugal force on the assumed two isotopes, which have equal atomic volumes, is balanced by the "osmotic pressure" which is set equal to $c R T$.

The problem can be attacked from another point of view. Suppose an isotopic liquid and its saturated vapor to be centrifuged side by side in a tube, the two phases being separated by a longitudinal membrane permeable only to the vapor. ${ }^{27}$ Let enough time be allowed for the at-

${ }^{24}$ In general, the peripheral pressure $P_{r}=\int_{r_{0}}^{r} \mathrm{~d} P=$

$$
\int \frac{\rho v^{2} \mathrm{~d} r}{r}=\int \rho \omega^{2} r \mathrm{~d} r:=\frac{\rho \omega^{2}\left(r^{2}-r_{0}^{2}\right)}{2}=\frac{\rho\left(v^{2}-v_{0}^{2}\right)}{2}
$$

if $\rho$, the density, is assumed constant, or an average value taken. Since $\Delta_{p} M$ is also proportional to $\left(v^{2}-v_{0}^{2}\right)$, the peripheral pressure is evidently directly proportional to the degree of separation obtained.

${ }^{25}$ It seems unlikely that this effect could interfere seriously with the method of evaporative centrifuging of a gas, already discussed.

${ }^{26}$ Poole, Phil. Mag., [6] 41, 818 (1.921).

${ }^{27}$ The vapor would of course be highly supersaturated and would tend to condense in the free vapor space at the peripheral end of the tube, thus leading to a distillation of the confined liquid until it was equally distributed on both sides of the membrane. 
tainment of a steady state. By the familiar type of proof based on the second law of thermodynamics, the (total) vapor pressure at every point must be that corresponding to equilibrium with the liquid under (centrifugal) pressure in the other tube. ${ }^{28}$ Now stuppose the equilibrium composition-distribution were not the same in both tubes, when allowed to reach equilibrium independently. Then there could not be composition equilibrium between the two phases across the membrane, when the other equilibria were satisfied. As a result of the conflicting equilibrium conditions, a perpetual circulation of the isotopes would go on (the denser ones in one direction, the less dense in the opposite, prestumably). No equilibrium could be attained in either phase, and the system would be capable of doing work continuously at constant temperature at the expense of thermal energy. This would violate the second law of thermodynamics, and the statistical mechanical principles 1nderlying it. We may then conclude that the isotopic composition will be identical at corresponding points in the two tubes. The equations previously obtained for an ideal gas are then applicable to any liquid whose saturated vapor behaves like an ideal gas, and undoubtedly also, by the principle of continuity, to any liquid or compressed gas whatever.

Experimental Work on the Separation of Isotopes by Liquid Centrifuging.-An unsuccessful attempt was made by Joly and Poole ${ }^{29}$ to detect a separation of the isotopes of lead after centrifuging ordinary lead in the liquid state in steel tubes, with a peripheral velocity of $10^{4} \mathrm{~cm}$. sec. The expected separation was, however, within the limit of error of the density determinations. They secured, nevertheless, a decided separation in the case of certain alloys. Poole ${ }^{26}$ later discussed the possibility of securing a separation with mercury, but concluded that the separation (30 parts per million in density) to be expected with their centrifuge would be too small to measure. Actually, much smaller changes in the density of mercury can be determined, as has been shown by Brönsted and Hevesy ${ }^{30}$ and especially by Mulliken and Harkins. ${ }^{2}$ With the idea of testing the theory experimentally, two steel tubes were made to fit a large laboratory centrifuge. Thick-walled glass tubes were first tried, but their capacity was small and breakage too frequent. A speed of about 2300 r.p.m. was attained. The inner end of each tube was $7.1 \mathrm{~cm}$. from the center of the centrifuge, the outer end $26.3 \mathrm{~cm}$. The tubes held 13 cc. each. The calculated extreme separation is $\left(\Delta_{p} M\right)=8.8$ parts This tendency, however, does not in the least invalidate the argument. It has no bearing on the equilibrium between the vapor and confined liquid at any point.

${ }^{28} \mathrm{As}$ far as the total vapor pressure is concerned, the isotopic composition of liquid or vapor should be a matter of indifference, since all the evidence shows that the vapor pressures of isotopes are practically, if not exactly, equal.

${ }^{29}$ Joly and Poole, Phil. Mag., [6] 39, 372 (1920).

${ }^{30}$ Brönsted and Hevesy, Phil. Mag., [6] 43, 31 (1922). 
per million in density. The centrifuged material was divided into thirds, and the densities of the inner and outer thirds compared. The expected difference was about $2 / 3 \times 8.8=5.9$ p.p.m. (by Equation 30). This is very much greater than the experimental error in the density determinations. The results were conclusively negative to within 0.5 p.p.m. in each of three runs (a 40-minute run with glass tubes, and two 8 -hour runs with the steel tubes). In the first of the latter runs there was a little vibration, but great care was taken in the second run to reduce this to a minimum by adjusting the weights of the tubes (these were somewhat unequal for the best balancing). There was still detectable vibration, however, which increased after the run had started. The pycnometer weighings, ${ }^{31}$ each corresponding to a separate refilling of the pycnometer, were made and corrected as described in the previous paper. They are all reproduced in Table I to show the conclusiveness of the results and to illustrate the accuracy of the density comparisons. In Run 1, the pycnometer used contained about $96 \mathrm{~g}$. of mercury, in the other runs, $107 \mathrm{~g}$.

TABI,F I

\begin{tabular}{|c|c|c|c|c|c|c|c|}
\hline \multirow[t]{2}{*}{ RuI } & Time & & \multicolumn{5}{|c|}{ Corrected weight of filled pycnometer } \\
\hline & Hours & & Uncentrifuged & & 'Heavy' Fraction & & "Light" Fractior \\
\hline \multirow[t]{3}{*}{1} & $2 / 3$ & & & & 116.32900 & & 116.32900 \\
\hline & & & & & 116.32885 & & 116.32897 \\
\hline & & & & Ay. & 116.32893 & Av. & 116.32899 \\
\hline \multirow[t]{3}{*}{2} & 7.5 & & 124.09339 & & 124.09343 & & 124.09339 \\
\hline & & & 124.09341 & & $(124.09321)$ & & 124.09338 \\
\hline & & & & & 124.09336 & & \\
\hline & & Av. & 124.09340 & Av. & 124.09340 & Av. & 124.09339 \\
\hline \multirow[t]{6}{*}{3} & 8 & & & & 124.09393 & & $\begin{array}{l}(124.09431) \\
(124,09355)\end{array}$ \\
\hline & & & & & 124.09308 & & $\begin{array}{l}(124.09355) \\
(124.09379)\end{array}$ \\
\hline & & & & & & & 124.09391 \\
\hline & & & & & & & 124.09400 \\
\hline & & & & & & & 124.09397 \\
\hline & & & & Av. & 124.09396 & Av. & 124.09396 \\
\hline
\end{tabular}

It was suspected that vibration was the cause of failure to obtain a separation. The process of establishment of the equilibrium state under centrifugal force is one of diffusion, ${ }^{32}$ so that the rate of diffusion is also an

${ }^{31}$ None of the weighings made has been omitted in the table. In taking averages, those in parentheses have been rejected. This is justified by the close agreement of the remaining values, as well as by other evidence (pump not working properly) in the case of the two low values for Sample 3. Low values are usually due to incomplete filling and are very likely to be wrong when consistent higher values are obtained. Uncommonly high values are usually due to an unncticed minute drop of mercury on the outside of the pycrometer.

${ }^{32}$ This factor is discussed in an article (of which only an abstract was available to the writer) by von Hevesy, Verh. Deutschen. Physik. Ges., 1,47 (1920), in connection with a discussion of Joly and Poole's atternpt (Ref. 29) to separate the lead isotopes. Von Hevesy also discusses the case of an aqueous solution of a lead salt. 
important factor. We may reasonably assume that the separation of the isotopes would take place at the same rate under centrifugal force as return to uniformity of composition would occur after the stopping of the centrifuge. We may assume $2.0 \mathrm{~g} . / \mathrm{cm} .{ }^{2} /$ day as a reasonable value for the autogenic diffusion of mercury. Then for a tube $20 \mathrm{~cm}$. long, if the difference of density at the two ends has any value $\Delta d$, we can obtain an idea of the rate of diffusion by assuming a uniform density gradient equal to $\Delta d / 20$. On this basis, it is found, assuming mercury to consist of a mixture of two isotopes differing by 3 units in atomic weight, that in 24 hours enough of the heavy isotopes would pass from one-half of the tube to the other to neutralize the initial gradient five times over, if the latter were maintained constant. From this rough calculation, it is evident that in 8 hours composition equilibrium under centrifugal force should be approached.

The effect of vibration and of diffusion-rate were directly tested, as follows. The lower half of each steel centrifuge tube was filled with some mercury having a density 12 p.p.m. greater (weight of filled pycnometer $124.09526 \mathrm{~g}$.) than that of ordinary mercury (weight of filled pycnometer $124.09401 \mathrm{~g}$.), which was used to fill the upper half of each tube. The tubes were then centrifuged at full speed for $1 / 2$ hour, there being slight vibration during the operation. The densities of the material in the two halves of the tube were now redetermined, and found practically identical (lower half, $W=124.09466$, upper half, $W=124.09461$ ). The tubes were then refilled in the same way with fresh material and allowed to stand quietly for the same total time as before ( 53 minutes). This time, the material of the lower half showed a decrease of density of only $0.15 \mathrm{mg}$. ( $W=124.09511$.) Finally, the same fractions were returned to the tubes which were then allowed to stand undisturbed for $81 / 2$ hours. Diffusion was now nearly complete. ${ }^{33}(W=124.09475$ for the material from the lower end of the tube; decrease $=0.51 \mathrm{mg}$. out of a possible $0.63 \mathrm{mg}$.)

Conclusions in Regard to Liquid Centrifuging.--The above results give direct proof that diffusion is sufficiently rapid to permit separation, but that vibration of the centrifuge is sufficient to prevent it (the effect of vibration would of course be less if diffusion were more rapid). The result shows that on account of the latter factor, the separation of isotopes by the centrifuging of a liquid is not a promising method, although it might be possible in a very accurately, heavily constructed and perfectly balanced centrifuge. The results do not, of course, give any evidence as

${ }^{33}$ Experiments of this sort can be used to determine the autogenic diffusion coefficient of mercury, somewhat as Gróh and von Hevesy [Ann. Physik, [4] 63, 85 (1920)] used a radioactive isotope to determine the corresponding coefficient in the case of lead. 
to the validity of the theory of centrifugal separation. The theoretical evidence for this, however, seems conclusive.

\section{Summary}

1. The theory of the separation of isotopes by thermal diffusion and by centrifuging is discussed. Equations are developed giving the difference in atomic weight obtainable in any operation, similar to the equations for diffusion and evaporation processes obtained in a previous paper. ${ }^{2}$

2 . For thermal diffusion, the difference in atomic weight between portions of an isotopic gas at temperatures $T_{1}$ and $T_{2}$, respectively is $\Delta_{t} M=$ $K \times \mathrm{B} \ln T_{1} / T_{2}$, approximately, the atomic weight being greater at the colder end. $B$ is the ordinary separation coefficient as defined in the previous paper. $K$ is an approximate constant for each element, having a value probably near $1 / 4$, and depending on the behavior of the molecule during impacts. The term $K B$ may be called the thermal separation coefficient. The method of thermal diffusion is shown to be much less effective as a means of separating isotopes than ordinary diffusion or evaporation. A somewhat more advantageous modification of the method is described under the name of evaporative thermal diffusion.

3. For the centrifuging of a gas the difference in atomic weight between the central and peripheral regions is $\Delta_{p} M=P\left(v^{2}-v_{0}^{2}\right)$, where $P$, the "centrifugal separation coefficient," is a characteristic constant for each element $\left(v\right.$ and $v_{0}$ denote velocities at the peripheral and central regions of the material under treatment). Values of $P$ for various elements are given. It is shown that the value of $P$ is unaffected by the state of combination of the element, even if the compound contains other isotopic elements. Thus the separation is equally great for all compounds of a given element. This is in contrast to the situation with all the other diffusion methods, for which the degree of separation of a given element in one operation is inversely proportional to the molecular weight of the compound. Further, the value of $P$ for any element is independent of the atomic weight, while the ordinary separation coefficient $B$ is inversely proportional to the latter. Hence, the theory is on this basis relatively increasingly more favorable to the centrifugal method as the atomic weight increases. $P$ is equal to $\frac{\left(M_{2}-M_{1}\right)^{2} x_{1} x_{2}}{2 R T}$ for a mixture of two isotopes, and for a mixture of several isotopes is given by $\frac{\Sigma_{a} \Sigma_{b} x_{a} x_{b}\left(M_{a}-M_{b}\right)^{2}}{2 R T} \cdot P$, unlike $B$, is inversely proportional to $T$, but depends on the atomic or molecular weight intervals $\left(M_{a}-M_{b}\right)$ and molfractions ( $x$ 's) in the same way as $B$.

4. Although for the heavy elements the theory predicts, for a peripheral velocity of $10^{5} \mathrm{~cm}$./sec. a separation many times that obtainable 
in a single diffusion or evaporation, it is shown that compression and condensation of the gas or vapor into the peripheral region make such large separations impracticable if carried out in any ordinary way. The pressure ratio between the two regions is given by $\frac{\ln p}{p_{0}}=\frac{M}{P} \cdot \frac{\Delta_{p} M}{2 R T}$ (strictly true only for an ideal gas), and so increases with atomic and molecular weight.

5. A special method which is called "evaporative centrifuging" is proposed whereby gas condensed in the periphery of the centrifuge at high speed would be allowed to evaporate very slowly, the light fraction being drawn off gradually at low pressure from the center of the apparatus. The process would be in effect precisely analogous to an evaporation in which the separation coefficient was increased from $B$ to $P v^{2}$. This method, applicable at room temperature to hydrogen chloride, bromide, selenide, telluride and silicide, and perhaps to other substances, though. less advantageously, with heating, might be expected with peripheral velocities up to $10^{5}$, to yield a separation 10 or 15 times as great in a single operation as would an ordinary diffusion or evaporation. No serious objection to the method is obvious. The method may be the most rapid method of separating isotopes for some of the elements of medium atomic weight, provided a suitable centrifuge of reasonable capacity and the necessary speed can be constructed. For the lighter or heavier elements, the method is less promising.

6. The theory of the separation of isotopes by the centrifuging of a liquid is discussed, and a thermodynamic demonstration given that the degree of separation for a given apparatus is identical for liquids, gases, and intermediate states of matter. An account is given of an attempt to test the theory in the case of liquid mercury. The conclusively negative results obtained are shown by an experiment to be attributable to a slight vibration of the centrifuge. This effect is likely to prove a limiting factor in any attempt to use the theoretically very promising method of liquid centrifuging. The effect of other factors is discussed, including that of diffusion rate. The latter is shown theoretically, and experimentally by determining the rate of interdiffusion of separated isotopes, to be sufficiently great in the case of mercury (and undoubtedly in general), to permit an approach to the theoretical equilibrium state of partial separation in a few hours.

7 . The above discussion applies to the separation by centrifuging of non-isotopic gases of nearly equal molecular weight $(e . g .$, air), and also of ideal solutions. The chief difficulty in the case of the latter would be the effect of vibration.

8. Some equations and discussions applicable to the previous paper are given, viz., Equations 19 and 19A, and References 17, 20, 22, and 34 . 
In particular, a demonstration is given of the at least approximate independence of the ordinary separation coefficient, for any element, of anything but the total molecular weight, even if the molecule contains other isotopic elements. Examples are also given in Table I of the extreme accuracy of the density comparisons which can be made with the pycnometer described in the previous paper.

It may be well to correct here certain conclusions given in the previous paper (pp. 40-41) in regard to the rate of separation of isotopes by gaseous diffusion, i.e., diffusion of an isotopic gas through a thin layer of another gas enclosed in the pores of a nnembrane. Briefly, this method is now found to be always less favorable than molecular diffusion, instead of more favorable, as previously concluded. This is due to the effect of the increased mean free path of the heavier molecules which results from persistence of velocity. This factor was expressly neglected before. Further, it now appears that mass motion, either positive or negative, should have practically no effect on the rate of separation, except indirectly as it may affect back pressure and so efficiency. A fuller discussion of this subject will be given later. Experimental work is now being carried on with mercury.

Acknowledgment shotild have been expressed in the previous paper of indebtedness to the C. M. Warren Fund of the American Academy of Arts and Sciences for a grant of five hundred dollars made to Professor William D. Harkins. A part of this grant was used in the purchase of an extremely accurate balance which was available for use in the previous investigation on the separation of the isotopes of mercury by the evaporation method, as well as in the present work.

CHICACO, II,IINOIS

Contribetion from the Wolcote Gibes Memorial, I,aboratory, Harvard UNIVERSITY]

\section{THE HEAT OF SOLUTION OF ZINC IN HYDROCHLORIC ACID}

\section{By TheODORE W. Richaf.ds AND THORBERgur ThORVAl,dSON Received March 1, 1922}

The heats of solution of metals in acids-data which are the basis of the computation of the heats of formation of all compounds of metalsare worthy of especial attention. The incompleteness of Thomsen's method of correcting his results for the heat of dilution of the solutions employed ${ }^{1}$ and the merely approximate character of early work renders revision desirable.

Revision was, indeed, begun over a decade ago. Values for the heat of solution of zinc, aluminum, magnesium, cadmium and iron in hydrochloric acicl were found which in some cases differed markedly from Thomsen's ' Richartis and Burgess, 'Thrs Jotranar, 32, 431 (1910). 